\title{
Fixação interna de pseudoartrose de escafoide com deformidade angular associada utilizando enxerto de ilíaco e placa volar: Série de oito casos
}

\section{Internal Fixation of Scaphoid Nonunion with Humpback Deformity Using Iliac Graft and Volar Plate: Series of 8 Cases}

\author{
Amanda Favaro Cagnolati ${ }^{1}$ Fernanda Ruiz Andrade ${ }^{1}{ }^{1}$ Luis Guilherme Rosifini Alves Rezende ${ }^{1 \odot}$ \\ Nilton Mazzer ${ }^{2 \odot}$ Raquel Metzker Mendes Sugano ${ }^{3 \odot}$ Larissa Martins Garcia ${ }^{3 \odot}$
}

\footnotetext{
1 Programa de Cirurgia da Mão, Departamento de Ortopedia e Anestesiologia, Hospital das Clínicas, Faculdade de Medicina de Ribeirão Preto, Universidade de São Paulo, Ribeirão Preto, SP, Brasil

2 Divisão da Cirurgia da Mão, Departamento de Ortopedia e Anestesiologia, Hospital das Clínicas, Faculdade de Medicina de Ribeirão Preto, Universidade de São Paulo, Ribeirão Preto, SP, Brasil

${ }^{3}$ Centro de Reabilitação, Hospital das Clínicas, Faculdade de Medicina de Ribeirão Preto, Universidade de São Paulo, Ribeirão Preto, SP, Brasil
}

Rev Bras Ortop 2021;56(5):588-593.

\begin{abstract}
Endereço para correspondência Amanda Favaro Cagnolati, MD, Departamento de Ortopedia e Anestesiologia, Hospital das Clínicas, Faculdade de Medicina de Ribeirão Preto, Universidade de São Paulo, Campus Universitário, Ribeirão Preto, SP, 14049-900, Brasil (e-mail: amandafavaro@terra.com.br).
\end{abstract}

\section{Resumo}

\section{Palavras-chave \\ - fixação interna \\ - pseudoartrose \\ - osso escafoide \\ - placas ósseas \\ - enxertos}

Objetivos A finalidade do presente artigo é avaliar os resultados clínicos, radiográficos e funcionais de pseudoartroses de escafoide com deformidade angular utilizando enxerto de ilíaco e placa volar.

Métodos Foram seguidos prospectivamente 8 pacientes, todos do sexo masculino, com idade média de 39,6 anos, com pseudoartrose de cintura de escafoide, com tempo médio de 19 meses de trauma sem cirurgias prévias. Os pacientes foram tratados com enxerto retirado da crista ilíaca e placa volar para escafoide. Os pacientes foram submetidos a avaliações radiográficas e tomográficas no pré-operatório e em 1 mês, 3 meses, 6 meses e 1 ano de pós-operatório. A altura carpal e os ângulos escafossemilunares e intraescafoides foram medidos no pré-operatório e aos 3 meses e 1 ano do pós-operatório. A função foi avaliada no pré-operatório e repetida em 1 ano pósoperatório através do escores Disabilities of the Arm, Shoulder, and Hand (DASH, na sigla em inglês) e Patient-related Wrist Evaluation (PWRE, na sigla em inglês), assim como pela aferição de força de pinças e de amplitude de movimento do punho e do polegar.

Resultados Houve consolidação em todos os casos, com tempo médio de 3,38 meses. Foram observadas duas complicações: uma infecção de ferida operatória do ilíaco e um parafuso da placa colocado no espaço escafossemilunar. Houve melhora nos recebido

16 de Dezembro de 2020

aceito

12 de Abril de 2021
DOI https://doi.org/

$10.1055 / \mathrm{s}-0041-1735830$ ISSN 0102-3616.

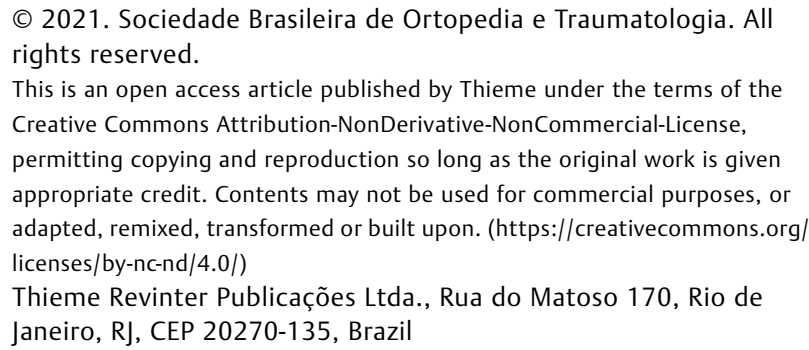

(c) 2021. Sociedade Brasileira de Ortopedia e Traumatologia. All rights reserved.

This is an open access article published by Thieme under the terms of the Creative Commons Attribution-NonDerivative-NonCommercial-License, permitting copying and reproduction so long as the original work is given appropriate credit. Contents may not be used for commercial purposes, or adapted, remixed, transformed or built upon. (https://creativecommons.org/ licenses/by-nc-nd/4.0/) Thieme Revinter Publicações Ltda., Rua do Matoso 170, Rio de Janeiro, RJ, CEP 20270-135, Brazil 


\begin{abstract}

\section{Keywords}

- internal fixation

- pseudarthrosis

- scaphoid bone

- bone plates

- transplants

Objectives The aim of the present article is a clinical and radiographic evaluation of scaphoid nonunion with humpback deformity using an iliac graft and a volar plate.

Methods Eight patients were followed-up prospectively, all male, with a mean age of 39.6 years old, with scaphoid waist nonunion, with an average of 19 months of trauma without previous surgery. The patients were treated with a graft removed from the iliac crest and a $1.5 \mathrm{~mm}$ blocked volar scaphoid plate (Medarthis AG, Basel, Switzerland). The patients were followed-up with radiographic evaluations and computed tomography $(\mathrm{CT})$. The patients were followed-up with radiographic evaluations and computed tomography (CT) scans in the preoperative period and 1 month, 3 months, 6 months and 1 year after the operation. The carpal height and the scaphosemilunar and intrascaphoid angles were measured preoperatively, 3 months, and 1 year postoperatively. The function was assessed preoperatively and repeated 1 year postoperatively using the Disabilities of the Arm, Shoulder, and Hand (DASH) and Patient-related Wrist Evaluation (PWRE scores), as well as by measurement of forceps clamp and wrist and thumb range of motion.

Results All cases were consolidated, with an average time of 3.38 months. Two complications were observed: an infection of the iliac surgical wound and a plaque element placed in the scapholunate space. Two patients opted to remove the implant because they had a painful click at maximum wrist flexion. There was improvement in the parameters of wrist and thumb range of motion and pinch strength in all measurements, with statistical significance for the PRWE and the scapholunate angle. Conclusion Although our sample was small, we achieved consolidation in all cases; the complications were expected in the literature and there were improvements in the radiographic patterns, in the functional assessment, and in the pain scale of our patients.
\end{abstract}

parâmetros de amplitude de movimento do punho e do polegar e de força de pinça em todas as avaliações funcionais, com significância estatística do PRWE e do ângulo escafossemilunar.

Conclusão Apesar da nossa amostra ter sido pequena, obtivemos consolidação em todos os casos operados, com complicações esperadas na literatura e melhoras tanto no padrão radiográfico quanto na avaliação funcional e na escala de dor dos nossos pacientes.

\section{Introdução}

As fraturas de escafoide são mais comuns em homens, e a faixa etária varia de 15 a 40 anos. Os maiores fatores de risco para pseudoartrose são fraturas desviadas, demora do paciente em procurar atendimento médico, ausência de diagnóstico (fraturas ocultas) e fraturas do polo proximal do escafoide, devido ao risco maior de diminuição do suprimento arterial deste fragmento. ${ }^{1,2}$ Pseudoartroses de escafoide podem ser definidas como estáveis ou não estáveis. Nas instáveis, é observado que as forças através do punho induzem a um movimento de flexão do fragmento proximal do escafoide, que assume uma deformidade em humpback ou "em corcova." A perda desta estabilização radial do carpo faz com que o punho colapse em um padrão de deformidade com o semilunar desviado dorsalmente na radiografia em perfil (DISI). ${ }^{1}$ As bordas dos fragmentos proximais e distais não consolidadas do escafoide atritam entre si e, após repetidos ciclos de carga, apresentam progressiva erosão e perda óssea. ${ }^{3}$
Tipicamente, o tratamento convencional de pseudoartroses de escafoide com perda óssea envolve o uso de enxerto ósseo corticoesponjoso colocado entre os fragmentos proximais e distais da não união, associado ou não a uma fixação interna. 0 enxerto pode ser retirado da crista ilíaca ou do terço distal do rádio e não há diferença na literatura em relação aos índices de consolidação. Primeiramente, houve a utilização de fios de Kirschner (fios de K) associados ao enxerto, depois a propagação de parafusos de compressão sem cabeça isolados ou em pares e, mais recentemente, o aparecimento de placas de suporte especificas para escafoide. ${ }^{4,5}$

Em 2016, surgiram trabalhos utilizando placas específicas para escafoide colocadas volarmente associadas a enxerto. Dodds et al. ${ }^{4}$ utilizaram em nove pacientes a placa associada a enxerto vascularizado pediculado baseado na artéria radial e demonstraram que a placa foi capaz de resistir às forças deformantes causadas pela fratura antiga do escafoide e 
segurou o enxerto colocado. Em agosto de 2016, Jurkowitsch et al. ${ }^{6}$ concluíram em estudos biomecânicos comparando placa e parafusos de compressão que a fixação com placa foi biomecanicamente superior a um parafuso isolado e que foi equivalente ao uso de dois parafusos de compressão.

Uma placa de $1,5 \mathrm{~mm}$ específica para escafoide foi utilizada na presente casuística de oito casos de pseudoartrose de escafoide com reabsorção óssea e deformidade em corcova, juntamente com enxerto estruturado corticoesponjoso de crista ilíaca. O objetivo foi avaliar a consolidação, a correção de parâmetros radiográficos e efetuar avaliações funcionais e de dor no período de 1 ano de seguimento pós-operatório.

\section{Material e Métodos}

O critério de inclusão foi pacientes com mínimo de seis 6 meses de fratura de escafoide sem cirurgia prévia que apresentavam perda óssea no sítio de não união, assim como deformidade em corcova, classificados segundo Alnot tipo IIB (pseudoartrose instável com defeito ósseo anterior e DISI).

Foram realizadas radiografias em anteroposterior (AP) e perfil do punho afetado e a mensuração dos ângulos escafossemilunar e intraescafoide e a medição da altura carpal na avaliação pré-operatória. O planejamento cirúrgico também incluiu radiografias do punho contralateral e tomografia computadorizada (TC) do punho afetado, realizados para assegurar o comprimento correto do enxerto a ser retirado e do tamanho do escafoide após a enxertia. ${ }^{4}$

A cirurgia foi realizada sob anestesia geral associada a bloqueio de plexo braquial no lado a ser operado com a colocação de um torniquete inflável superiormente neste braço. A via de acesso foi a clássica anterior de Russe, onde as bordas do escafoide foram curetadas até osso viável ser encontrado, retirando possíveis tecidos fibrosos interpostos, e o defeito volar foi medido após a abertura do foco de pseudoartrose, facilitado pela utilização complementar de fios de $\mathrm{K}$ de $1,0 \mathrm{~mm}$ em cada fragmento ósseo atuando como joysticks.

$O$ intensificador de imagens foi utilizado para certificar a correção apropriada da deformidade do escafoide. A rotação do semilunar foi corrigida com a inserção de um fio de K de $1,5 \mathrm{~mm}$ fixando o rádio e o semilunar. Após a medição do defeito ósseo, realizou-se a infiltração da região ilíaca contralateral do paciente com xilocaína a $2 \%$ com vasoconstritor, incisão sobre a crista ilíaca, e a retirada do enxerto de ilíaco com serra oscilatória, criando uma cunha tricortical com enxerto corticoesponjoso no centro e com mensuração ligeiramente superior à medida. Enxerto esponjoso também foi retirado e posteriormente colocado junto à placa.

A placa utilizada foi uma placa de perfil baixo bloqueada para escafoide APTUS Hand (Medarthis AG, Basel, Switzerland). aplicada na face volar do escafoide, mantida centralizada na fluoroscopia na posição AP, e foi iniciada a fixação com dois parafusos corticais, um em cada fragmento. Três parafusos foram colocados em cada fragmento ósseo e não houve a necessidade de parafusos bloqueados de rotina, uma vez que o estoque ósseo dos pacientes estudados era de boa qualidade (-Figura $\mathbf{1}$ ).

Os pacientes permaneceram com imobilização gessada axilopalmar com inclusão do polegar por 2 semanas no pós-

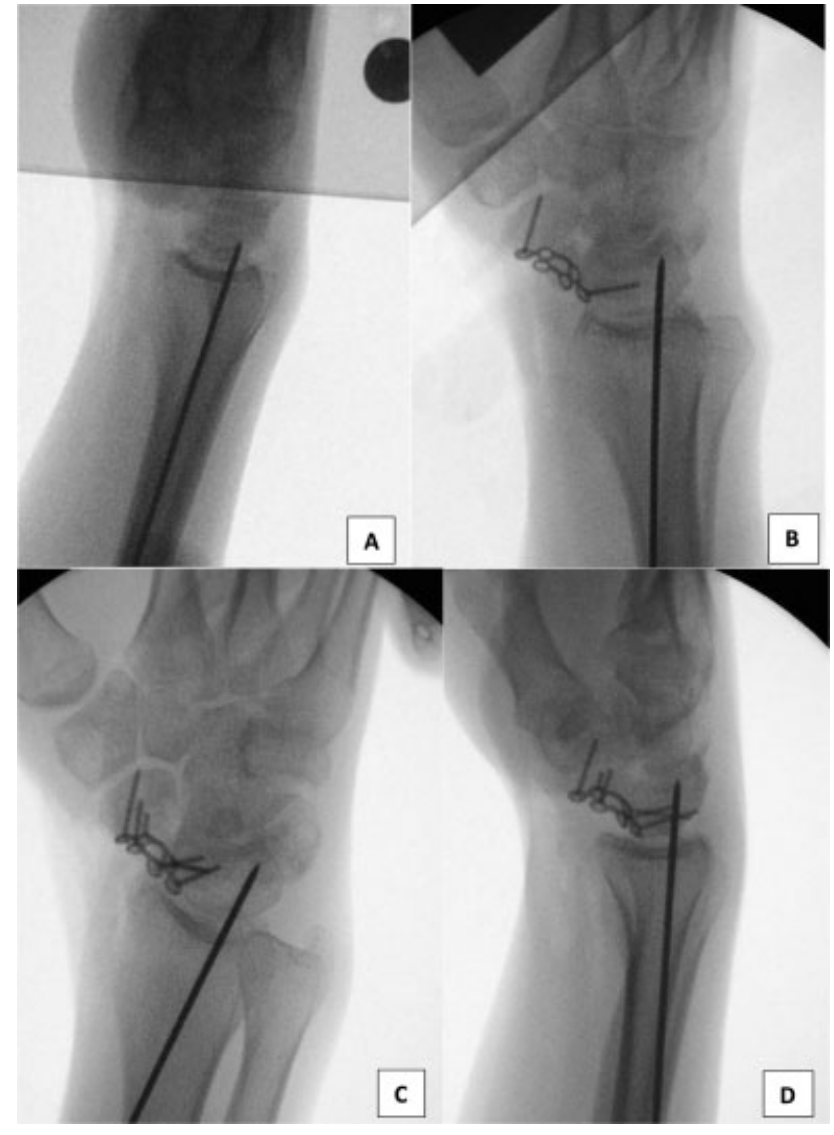

Fig. 1 Imagens da fluoroscopia intraoperatória. (A) Correção da extensão do semilunar utilizando um fio de K. (B) Colocação de um parafuso cortical em cada fragmento. (C) Incidência oblíqua para confirmação correta do tamanho dos parafusos. (D) Radiografia em perfil final demonstrando placa na posição correta, enxerto coaptado e tamanhos ideais dos parafusos.

operatório. Após a retirada dos pontos, houve a transição para uma órtese, a qual foi mantida até que houvesse critérios de consolidação nas radiografias pós-operatórias e nas tomografias controles. Os critérios utilizados para a detecção de consolidação foram: ausência de dor, evidência radiográfica de ponte óssea através do enxerto (> 50\% de trabéculas ósseas atravessando os fragmentos na TC) e ausência de sinais de soltura do implante. ${ }^{3}$

O seguimento ambulatorial foi de 2 semanas (retirada de pontos e troca de imobilização), 4 semanas (retirada do fio de K), 3 meses, 6 meses e 1 ano pós-operatório. As radiografias foram realizadas com 4 semanas, 3 meses, 6 meses e 1 ano pós-operatórios. A TC foi realizada no $3^{\circ}$ mês e repetida a cada 3 meses caso houvesse dúvida quanto à consolidação.

Foram realizadas avaliações junto à equipe de fisioterapia no pré-operatório e com 3, 6 e 12 meses de pós-operatório. Foi excluída do estudo a avaliação com 6 meses de pósoperatório, frente ao não comparecimento de alguns pacientes. As avaliações foram: teste de força das pinças do tipo lateral com dinamômetro hidráulico (PinchGauge), avaliação da amplitude de movimento ativa do polegar e punho (flexão, extensão, abdução radial e palmar do polegar; flexão, extensão, desvios radiais e ulnar, pronação e supinação do punho), escala visual analógica (EVA) de dor (repouso e 
movimento) e os questionários Disabilities of the Arm, Shoulder, and Hand (DASH, na sigla em inglês) e Patientrelated Wrist Evaluation (PRWE).

Devido ao tamanho pequeno da amostra, os dados foram descritos usando mediana, valores mínimos e máximos e desvio padrão (DP). Na avaliação fisioterápica de 2 momentos, no pré-operatório e com 1 ano de pós-operatório, foi utilizado o teste de Wilcoxon, e nos parâmetros radiográficos comparando o pré-operatório, 3 meses e 1 ano pósoperatórios, foi utilizado o teste $t$ de Student. A significância estatística foi aceita quando $p \leq 0,05$.

\section{Ética}

O presente trabalho foi aprovado pelo comitê de ética em pesquisa, de acordo com a resolução CNS 466/2012, em 07 de agosto de 2017, parecer número 2.204.444.

\section{Resultados}

Foram submetidos a cirurgia oito pacientes do sexo masculino, sendo sete pacientes com pseudoartrose de escafoide da mão direita e um da mão esquerda, todos membros dominantes. A idade média dos pacientes foi de 39,6 anos (27 a 65 anos).

O tempo médio desde o trauma até a cirurgia foi de 19 meses ( 6 a 24 meses).

A consolidação óssea foi obtida em $100 \%$ dos pacientes, com tempo médio de 3,38 meses (apenas 1 paciente obteve consolidação radiográfica definida aos 6 meses de pósoperatório, os demais a obtiveram aos 3 meses). Todos os pacientes realizaram tomografia com 3 meses de pós-operatório e 1 paciente a repetiu com 6 meses após a cirurgia por dúvidas em relação à consolidação.

Um paciente apresentou como complicação dor importante e notou-se a presença de um parafuso proximal da placa no intervalo escafossemilunar. Este foi submetido à retirada da placa com 4 meses de pós-operatório, quando já havia sinais tomográficos de consolidação. Um paciente apresentou infecção aguda (28 dias pós-operatórios) da ferida operatória na região do ilíaco e foi submetido a desbridamento cirúrgico e antibioticoterapia guiada por culturas, com cicatrização da ferida.

Nos parâmetros radiográficos, o alinhamento carpal, como indicado pela mensuração do ângulo escafossemilunar, foi alterado após a cirurgia de $65^{\circ}$ (DP 14.8) para $40^{\circ}$ (DP 40.2) com $p=0,006$. Todos os parâmetros de avaliações funcionais comparando o pré-operatório e o pós-operatório de 1 ano melhoraram: a abdução radial do polegar subiu de uma média de $49,63^{\circ}$ para $56^{\circ}$; o desvio radial médio subiu de $12^{\circ}$ para $14^{\circ}$; o desvio ulnar médio subiu de $23^{\circ}$ para $28^{\circ}$; a flexão média do punho subiu de $53^{\circ}$ para $61^{\circ}$; e a extensão média do punho subiu de $37^{\circ}$ para $46^{\circ}$. Não houve alterações nas avaliações de pinças e na flexão e extensão da articulação metacarpofalangeana do polegar. Nas avaliações de dor em repouso, os parâmetros decresceram de 3 para 1,5, e os de dor ao movimento decresceram de 8 para 5,3. 0 quickdash decresceu de 56 para 34 e o PRWE de 68 para 31, com $p=0,0190$ (-Tabelas 1 e 2).

\begin{tabular}{|c|c|c|c|c|c|c|c|c|c|}
\hline$\sum_{\substack{\alpha \\
\Sigma}}^{\mathrm{u}}$ & in & $\stackrel{\infty}{\sim}$ & เน & $\infty$ & $\begin{array}{l}n \\
\infty \\
\infty\end{array}$ & \begin{tabular}{|l} 
ก \\
ผn \\
ผn
\end{tabular} & $\zeta$ & $\begin{array}{l}\qquad \\
\tilde{6}\end{array}$ & \\
\hline 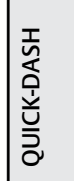 & \begin{tabular}{l} 
N \\
\multirow{N}{n}{}
\end{tabular} & $\infty$ & $\stackrel{\nabla}{m}$ & $\mid \begin{array}{c}m \\
\infty\end{array}$ & 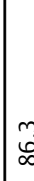 & $\stackrel{m}{\stackrel{n}{\sim}}$ & $\stackrel{\text { レ }}{\forall}$ & 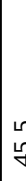 & \\
\hline 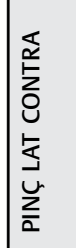 & $\stackrel{\infty}{\sim}$ & $\approx$ & $\begin{array}{c}m \\
\sigma\end{array}$ & $\stackrel{n}{2}$ & 7 & ñ & ڤ & $\hat{\sigma}$ & \\
\hline 嵅上 & $\stackrel{0}{r}$ & $\stackrel{m}{a}$ & $\begin{array}{l}m \\
\text { L }\end{array}$ & $\circ$ & 兄 & $\ln ^{n}$ & $\hat{\sigma}$ & z & \\
\hline 要求 & $a$ & in & $\sigma$ & $\stackrel{0}{\circ}$ & 잉 & $\sigma$ & $\infty$ & $\sigma$ & \\
\hline 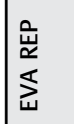 & 0 & $\sim$ & $m$ & เn & เ & $r$ & 0 & 0 & \\
\hline 늠ㅉㅁ & $\bullet$ & $\stackrel{\llcorner}{m}$ & $\stackrel{\circ}{-}$ & 8 & $\%$ & $\stackrel{\searrow}{\sim}$ & $\stackrel{\searrow}{\nearrow}$ & $\vartheta$ & \\
\hline 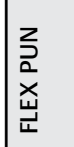 & প & $\stackrel{\text { ำ }}{\sigma}$ & 8 & $\infty$ & $\mathscr{q}$ & $\stackrel{\ddagger}{\sim}$ & เั & $\stackrel{\text { }}{\complement}$ & \\
\hline 㟔焉 & $\stackrel{\infty}{m}$ & $\stackrel{\llcorner}{\sim}$ & $\stackrel{\sim}{~}$ & $\stackrel{\Perp}{\sim}$ & 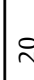 & 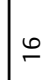 & 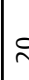 & 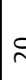 & \\
\hline $\mid \begin{array}{l}\frac{a}{\alpha} \\
\text { 㟧 } \\
\text { a }\end{array}$ & શ & $\stackrel{ }{-}$ & $\stackrel{ㅇ}{\leftarrow}$ & i & શ & ㄱ. & 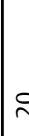 & $\subsetneq$ & \\
\hline 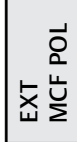 & 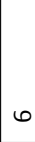 & 0 & 0 & 0 & શ & 0 & $c$ & $c$ & \\
\hline 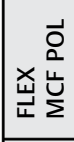 & $\stackrel{\circ}{\wedge}$ & ㅇํ & ำ & 8 & \% & 8 & பீ & $\varepsilon$ & \\
\hline 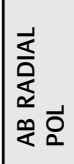 & 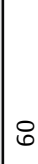 & ㅇํ & 우 & ص & $\stackrel{ \pm}{\sim}$ & $\stackrel{\infty}{+}$ & 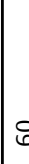 & 5 & \\
\hline 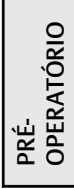 & - & $\sim$ & $m$ & $\nabla$ & in & 0 & $r$ & 0 & \\
\hline
\end{tabular}




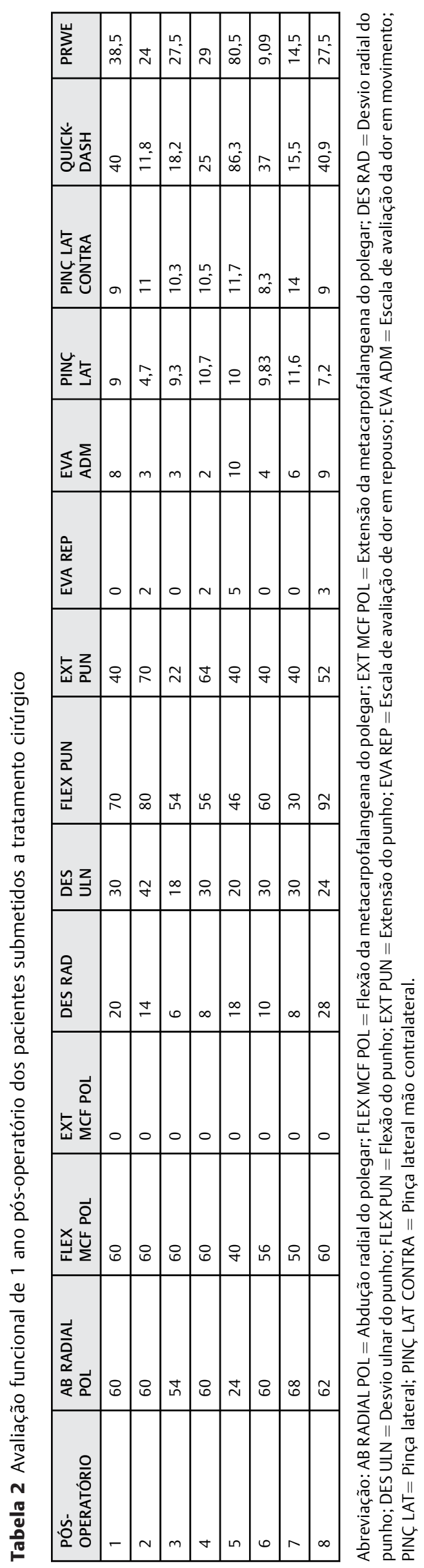

\section{Discussão}

O tratamento da pseudoartrose de escafoide com placas no fragmento proximal e um parafuso no fragmento distal foi descrito por Huene et al. ${ }^{7}$, que citou a sua utilização nos casos mais difíceis, nos quais a fixação com parafuso não era ideal. 0 uso da placa é idealmente reservado aos casos com deformidade em corcova do escafoide e aos casos já operados sem sucesso nos quais não há espaço para o uso de um parafuso isolado. A placa atua como um batente segurando o enxerto no local, evitando sua extrusão e anulando as forças de flexão que atuam sobre a cintura do escafoide. ${ }^{8}$

A literatura relata taxas de consolidação de 71 a $95 \%$ utilizando enxertos variados e parafusos de compressão sem cabeça tipo Herbert. Ghoneim, ${ }^{3}$ em 2011, reportou consolidação em 93\% dos 14 casos operados com placa Medarthis de $1,5 \mathrm{~mm}$. Dodds et al. ${ }^{4,8}$ conseguiram uma taxa de consolidação de $87 \%$, com 8 dos 9 casos operados com enxerto vascularizado de rádio e placa para escafoide, pacientes estes com falha inicial de um tratamento prévio. Em 2017, os mesmos autores aumentaram esta casuística para 20 casos, obtendo consolidação em 18 casos (90\%), com melhora da função e dor, demonstrando que esta cirurgia foi uma alternativa a procedimentos de salvamento, em pacientes reoperados. Esteban-Feliu et al., ${ }^{9}$ em 2017, seguiram 15 pacientes por 3 anos e obtiveram $87 \%$ de consolidação. 0 seguimento do presente estudo foi de 12 meses e obteve-se 100\% de consolidação nos 8 casos operados (-Figura 2).

$O$ fato de a placa ser de suporte volar e colocada intrarticularmente pode causar problemas. Tecnicamente, no intraoperatório, é recomendada a obtenção de inúmeras imagens de fluoroscopia em posições oblíquas para determinar a redução escafoide, a localização do enxerto e a posição da placa e dos parafusos. A penetração dos parafusos intra-articulares pode ser difícil de evitar, principalmente na radioescafoide e distalmente na escafotrapeziotrapezóide. A retirada da placa também é recomendada entre 9 a 12 meses após a cirurgia em pacientes que têm impacto à flexão máxima do punho, apresentando um estalido doloroso durante a flexão do punho. ${ }^{8,9}$ Foi necessária a retirada de uma placa devido a um parafuso se encontrar no espaço escafossemilunar e de duas devido aos pacientes terem apresentado dor e limitação na flexão do punho.

Em estudos mais recentes, de 2019, Burgos et al., apesar de conseguirem $100 \%$ de consolidação em sua série de 8 casos também operados com enxerto de crista ilíaca e placas volares, relatam diminuição do arco de movimento e da força de preensão em sua avaliação de 12 semanas de pós-operatório. ${ }^{10} \mathrm{Na}$ avaliação funcional do presente estudo, realizada com 1 ano de pós-operatório, houve melhora de todos os parâmetros avaliados e do PRWE, com melhora significativa, caindo de 68 para $31(p=0,01)$.

O sucesso de nossos casos não se deve exclusivamente ao uso da placa. Fatores como tempo relativamente curto de pseudoartrose (19 meses), pacientes jovens e sem comorbidades e com bom estoque ósseo certamente influenciaram no sucesso da consolidação. A presente série de casos possui limitações, como o pequeno número amostral, e ressaltamos 


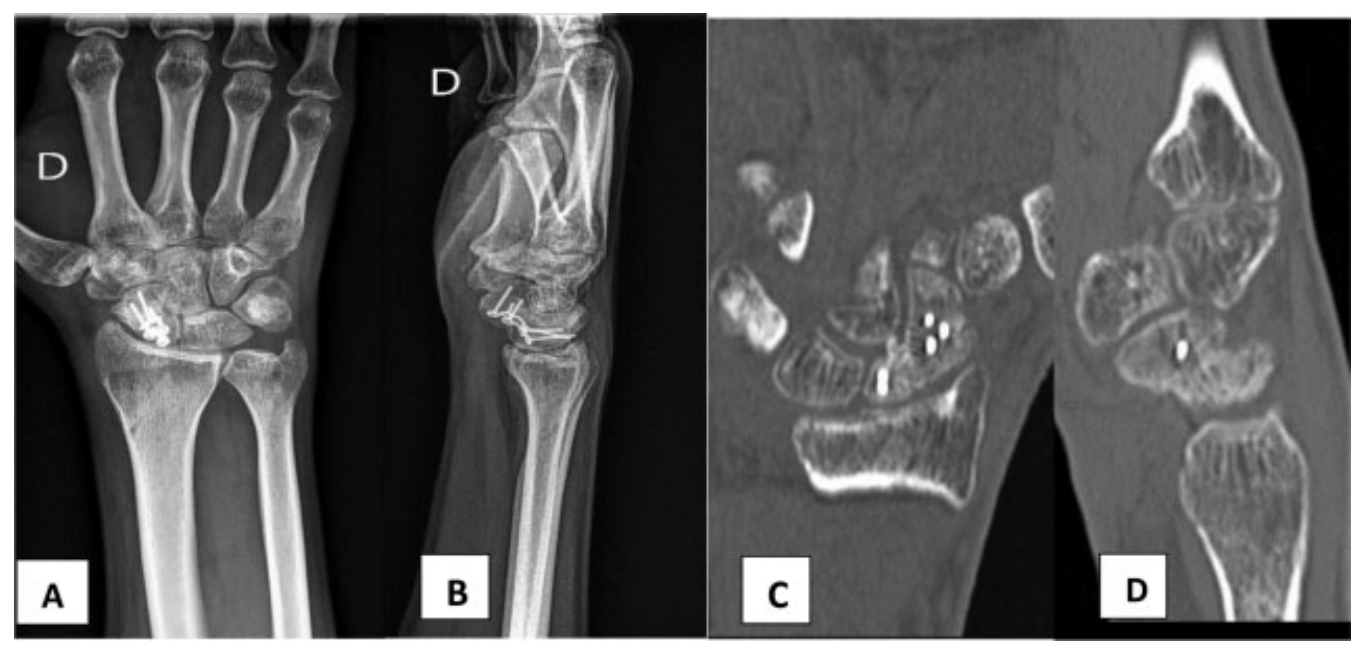

Fig. 2 Paciente com 3 meses de pós-operatório. (A) Radiografia em anteropoasterior demonstrando placa bem colocada e pseudoartrose consolidada. (B) Radiografia em perfil demonstrando total integração entre os fragmentos (C) Corte frontal da tomografia computadorizada demonstrando ponte óssea entre os fragmentos. (D) Perfil tomográfico demonstrando consolidação da pseudoartrose.

a necessidade de um estudo comparativo com utilização de parafuso sem cabeça. Devido à dificuldade de disponibilização e ao alto custo do material comparado com a grande disponibilidade e diversidade de parafusos sem cabeça para escafoide, compreendemos que esta técnica dificilmente será utilizada a curto prazo e de rotina; porém, ela pode ser uma opção em casos selecionados e nas pseudoartroses recalcitrantes, com falha de consolidação em cirurgia prévia.

\section{Conclusão}

A utilização da placa associada a enxerto de ilíaco nas pseudoartroses de escafoide com deformidade angular melhorou os parâmetros de alinhamento carpal dos pacientes, os parâmetros de mobilidade do punho e de dor comparados com os parâmetros pré-operatórios, com aumento do DASH, apresentando índices de consolidação e de complicações semelhantes aos descritos na literatura. Ressalta-se a necessidade de mais estudos, com grupo controle utilizando parafusos tipo Herbert e uma maior disponibilidade de acesso ao material utilizado.

Nota

Trabalho desenvolvido no Departamento de Ortopedia e Anestesiologia, Hospital das Clínicas, Faculdade de Medicina de Ribeirão Preto, Universidade de São Paulo (HCFMRP-USP), Ribeirão Preto, SP, Brasil

\section{Suporte Financeiro}

Não houve suporte financeiro de fontes públicas, comerciais, ou sem fins lucrativos.
Conflito de Interesses

Os autores declaram não haver conflito de interesses.

\section{Referências}

1 Bindra R, Bednar M, Light T. Volar wedge grafting for scaphoid nonunion with collapse. J Hand Surg Am 2008;33(06):974-979

2 Buijze GA, Ochtman L, Ring D. Management of scaphoid nonunion. J Hand Surg Am 2012;37(05):1095-1100

3 Ghoneim A. The unstable nonunited scaphoid waist fracture: results of treatment by open reduction, anterior wedge grafting, and internal fixation by volar buttress plate. J Hand Surg Am 2011; 36(01):17-24

4 Dodds SD, Halim A. Scaphoid Plate Fixation and Volar Carpal Artery Vascularized Bone Graft for Recalcitrant Scaphoid Nonunions. J Hand Surg Am 2016;41(07):e191-e198

5 Cooney WP, Linscheid RL, Dobyns JH, Wood MB. Scaphoid nonunion: role of anterior interpositional bone grafts. J Hand Surg Am 1988;13(05):635-650

6 Jurkowitsch J, Dall'Ara E, Quadlbauer S, et al. Rotational stability in screw-fixed scaphoid fractures compared to plate-fixed scaphoid fractures. Arch Orthop Trauma Surg 2016;136(11): 1623-1628

7 Huene DR, Huene DS. Treatment of nonunions of the scaphoid with the Ender compression blade plate system. J Hand Surg Am 1991;16(05):913-922

8 Dodds SD, Williams JB, Seiter M, Chen C. Lessons learned from volar plate fixation of scaphoid fracture nonunions. J Hand Surg Eur Vol 2018;43(01):57-65

9 Esteban-Feliu I, Barrera-Ochoa S, Vidal-Tarrason N, Mir-Simon B, Lluch A, Mir-Bullo X. Volar Plate Fixation to Treat Scaphoid Nonunion: A Case Series With Minimum 3 Years of Follow-Up. J Hand Surg Am 2018;43(06):569.e1-569.e8

10 Burgos FH, Nakamoto JC, Nakamoto HA, Iwase FDC, Mattar R. Treatment of scaphoid nonunion with volar locked plate. Acta Ortop Bras 2019;27(03):141-145 